Pacific Journal of Mathematics

THE GENERALIZED TRANSLATIONAL HULL OF A 


\title{
THE GENERALIZED TRANSLATIONAL HULL OF A SEMIGROUP
}

\author{
JOHN K. LUEDEMAN
}

\begin{abstract}
For a pair $(\mathscr{R}, \mathscr{L})$ consisting of a right quotient filter $\mathscr{R}$ and left quotient filter $\mathscr{L}$ on the semigroup $S$, a translational hull $\Omega(S: \mathscr{R}, \mathscr{L})$ is constructed. The results of Grillet and Petrich hold for $\Omega(S: \mathscr{R}, \mathscr{L})$.

Specializing $\mathscr{R}$ and $\mathscr{L}$ one obtains the usual translational hull $\Omega(S)$ of $S$ and the semigroup of quotients $Q(S)$ of $S$ due to Hinkle and McMorris. These results are applied to a weakly reductive semigroup $S$ to show that $\Omega(S)=\Omega\left(S^{n}\right)$ for any positive integer $n$.
\end{abstract}

In recent years two seemingly unconnected developements have occurred in the theory of semigroups. Grillet and Petrich [4] have studied ideal extensions of a semigroup $S$ by means of a universal extension $\Omega(S)$ of $S$, the translational hull of $S$. On the other hand, McMorris [7] and Hinkle [5] have developed a theory of one-sided semigroups of quotients of $S$ using a maximal semigroup $Q(S)$ of quotients. Under certain conditions $Q(S)$ is essential over $S$ while under similar conditions $\Omega(S)$ is a congruence dense extension of $S$. Berthiaume [1] showed that congruence dense extension and essential extension are the same concept. This similarity, along with many others, between $Q(S)$ and $\Omega(S)$ leads one to suspect the existence of a theory of semigroup extensions more general than the above mentioned theories. In this paper we offer a candidate for a general theory. In section three we show that our concept might reasonably be called a two-sided semigroup of quotients, having given our constructions in sections one and two. In section five we show that our construction yields an essential extension of $S$ maximal in a certain category. In section four, we follow the lead of Grillet and Petrich [4] and examine extensions of $S$ which are somewhat weaker than essential extensions. Along the way we obtain as corollaries some results of Hinkle [5] and Grillet and Petrich [4].

1. Basic definitions. In this paper, $S$ will be a semigroup with zero, denoted by 0 .

A left $S$-set ${ }_{s} K$ is a set $K$, with a distinguished element $\mathcal{O}$, having a scalar multiplication $S \times K \rightarrow K$ satisfying for all $s, t \in S$ and $k \in K,(s t) k=s(t k)$, and $0 s=\mathcal{O}$ and $k \mathscr{O}=\mathcal{O}$.

Similarly one can define a right $S$-set $K_{S}$ (with $O$ ).

In this paper we will not distinguish between $\mathcal{O}$, the zero of $K$ 
and 0 , the zero of $S$. The meaning of the symbol 0 will be clear from the context.

An $(S, S)$-set ${ }_{S} K_{S}$ is a set $K$ with scalar multiplications $S \times K \rightarrow K$ and $K \times S \rightarrow K$ such that ${ }_{s} K$ is a left $S$-set, $K_{S}$ is a right $S$-set, and for $s, t \in S$ and $k \in K, s(k t)=(s k) t$.

A homomorphism $\rho:{ }_{S} K \rightarrow{ }_{S} M$ of left $S$-sets is a mapping $\rho: K \rightarrow M$ satisfying $(s k) \rho=s(k \rho)$ for all $s \in S$ and $k \in K$.

Similarly one defines "homomorphism of right $S$-sets" and "homomorphism of $(S, S)$-sets". We write left $S$-homomorphisms on the right of their argument and right $S$-homomorphisms on the left.

${ }_{s} K$ is a sub $S$-set of ${ }_{s} M$ if $K \subseteq M$ and $K$ is an $S$-set under the operation $S \times M \rightarrow M$.

Definition (Hinkle [5]). A collection $\mathscr{R}$ of right ideals of $S$ is a right quotient filter on $S$ if

(1) if $A, B$ are right ideals of $S, A \subseteq B$ and $A \in \mathscr{R}$, then $B \in \mathscr{R}$

(2) if $A, B \in \mathscr{R}$ and $\lambda: A \rightarrow S$ is a right $S$-set homomorphism, then $\lambda^{-1}(B)=\{a \in A \mid \lambda a \in B\} \in \mathscr{R}$.

Hinkle has shown that a right quotient filter is closed under finite intersections and if $A \in \mathscr{R}$ and $s \in S$, then

$$
s^{-1} A=\{t \in S \mid s t \in A\} \in \mathscr{R} .
$$

An extension $M_{S}$ of $K_{S}$ is an $\mathscr{R}$-extension if for $m \in M$,

$$
m^{-1} K=\{s \in S \mid m s \in K\} \in \mathscr{R} .
$$

Dually one can define a left quotient filter $\mathscr{L}$ on $S$ and " $\mathscr{L}$ extension."

An $(S, S)$-set ${ }_{S} V_{S}$ is an $(\mathscr{R}, \mathscr{L})$-extension of ${ }_{S} K_{S}$ if $V_{S}$ is an $\mathscr{R}$ extension of $K_{S}$ and ${ }_{S} V$ is an $\mathscr{L}$-extension of ${ }_{s} K$.

A right quotient filter $\mathscr{R}$ is idempotent if whenever $A \in \mathscr{R}, I$ is a right ideal of $S$ and $a^{-1} I \in \mathscr{R}$ for all $a \in A$, then $I \in \mathscr{R}$.

This condition is equivalent to the condition: if $A \in \mathscr{R}$ and for each $a \in A$ there is $R_{a} \in \mathscr{R}$, then

$$
\bigcup_{a \in A} a R_{a} \in \mathscr{R} \text {. }
$$

2. The construction. Let $\mathscr{L}$ be a left quotient filter on $S$, $\mathscr{R}$ be a right quotient filter on $S$, and $K$ be an $(S, S)$-set. Consider all pairs $(\lambda, \rho)$ where $\lambda: D_{\lambda} \rightarrow K$ is a right $S$-homomorphism with domain $D_{\lambda} \in \mathscr{R}$ and $\rho: D_{\rho} \rightarrow K$ is a left $S$-homomorphism with domain $D_{\rho} \in \mathscr{L}$.

Definition 2.1. The pair $(\lambda, \rho)$ is $(\mathscr{R}, \mathscr{L})$-linked if for all 
$y \in D_{\lambda}$ and $x \in D_{\rho}, x(\lambda y)=(x \rho) y$.

Let $B(K: \mathscr{R}, \mathscr{L})$ be the collection of all such $(\mathscr{R}, \mathscr{L})$-linked pairs. Notice that for $k \in K$, the maps $\lambda_{k}: S \rightarrow K$ defined by $\lambda_{k}(s)=k s$ and $\rho_{k}: S \rightarrow K$ defined by $(s) \rho_{k}=s k$ given an $(\mathscr{R}, \mathscr{L})$-linked pair $\left(\lambda_{k}, \rho_{k}\right) \in B(K: \mathscr{R}, \mathscr{L})$. Moreover, $B(K: \mathscr{R}, \mathscr{L})$ is an $(S, S)$-set under the operation $s(\lambda, \rho)=(s \lambda, s \rho)$ where $s \lambda: D_{\lambda} \rightarrow K$ is given by $s \lambda(t)=$ $s[\lambda(t)]$ and $s \rho: D_{s \rho} \rightarrow K$ is given by $(t) s \rho=(t s) \rho$ where $D_{s \rho}=\left(D_{\rho}\right) s^{-1} \in \mathscr{L}$. $(s \lambda, s \rho)$ is linked since for $y \in D_{\lambda}$ and $x \in D_{s \rho}$,

$$
\begin{aligned}
x((s \dot{\lambda}) y) & =x[s(\lambda y)]=(x s)(\lambda y)=[(x s) \rho] y \\
& =[x(s \rho)] y
\end{aligned}
$$

since $x s \in D_{\rho}$ and $(\lambda, \rho)$ is linked. The definition of $\rho s$ and $\lambda s$ and the multiplication $(\lambda, \rho) s=(\lambda s, \rho s)$ is similar.

Where $K=S$, then $B(K: \mathscr{R}, \mathscr{L})$ is a partial transformation semigroup.

Define a relation $\Theta$ on $B(K: \mathscr{R}, \mathscr{L})$ by $(\lambda, \rho) \Theta\left(\lambda^{\prime}, \rho^{\prime}\right)$ iff

(1) there is $R \in \mathscr{R}$ with $R \subseteq D_{\lambda} \cap D_{\lambda^{\prime}}$ and $\lambda r=\lambda^{\prime} r$ for all $r \in R$, and $t \in L$.

(2) there is $L \in \mathscr{L}$ with $L \cong D_{\rho} \cap D_{\rho}$, and $t \rho=t \rho^{\prime}$ for all

Lemma 2.2. $\Theta$ is an $(S, S)$-congruence on $B(K: \mathscr{R}, \mathscr{L})$.

COROLLARY 2.3. $\Theta$ is also a semigroup congruence on $B(S: \mathscr{R}, \mathscr{L})$.

The straightforward proof of the above lemma and its corollary will be omitted.

The quotient $(S, S)$-set $B(K: \mathscr{R}, \mathscr{L}) / \Theta$ will be denoted by $\Omega(K: \mathscr{R}, \mathscr{L})$ and is called the $(\mathscr{R}, \mathscr{L})$-translational hull of $K$.

We usually denote the class of $(\lambda, \rho)$ in $\Omega(K: \mathscr{R}, \mathscr{L})$ by $(\lambda, \rho)$, but when clarification is needed, we denote it by $[\lambda, \rho]$.

There is a canonical $(S, S)$ homomorphism $\pi$ of $K$ into $\Omega(K: \mathscr{R}, \mathscr{L})$ given by $\pi(k)=\left(\lambda_{k}, \rho_{k}\right)$. When $K=S, \pi$ is a semigroup homomorphism.

DeFinition 2.4. If the homomorphism $\pi: K \rightarrow \Omega(K: \mathscr{R}, \mathscr{L})$ is injective, $K$ is said to be $(\mathscr{R}, \mathscr{L})$-reductive.

REMARKs. (1) When $\mathscr{L}$ is the collection of all left ideals of $S$, then $\Omega(S: \mathscr{R}, \mathscr{L})$ is semigroup isomorphic to $Q_{\mathscr{R}}(S)$, the semigroup of right quotients of $S$ developed by Hinkle [5]. 
Proof. The map $\sigma: \Omega(S: \mathscr{R}, \mathscr{L}) \rightarrow Q_{\mathscr{R}}(S)$ given by $\sigma[\lambda, \rho]=[\lambda]$ is the desired isomorphism. Since $\sigma[\lambda, \rho]=\sigma\left[\lambda^{\prime}, \rho^{\prime}\right]$ implies $[\lambda]=\left[\lambda^{\prime}\right]$, $\lambda$ and $\lambda^{\prime}$ agree on some $R \in \mathscr{R}$ and so $[\lambda, \rho]=\left[\lambda^{\prime}, \rho^{\prime}\right]$ since $\rho$ and $\rho^{\prime}$ agree on $(0) \in \mathscr{L}$ and so $\sigma$ is injective. Moreover for $[\lambda] \in Q_{\mathscr{R}}(S)$, $\sigma\left[\lambda, 1_{S}\right]=[\lambda]$ where $1_{S}: S \rightarrow S$ is the identity map on $S$ and $\sigma$ is surjective.

To see that $\sigma$ is a homomorphism, let $[\lambda, \rho],\left[\lambda^{\prime}, \rho^{\prime}\right] \in \Omega(S: \mathscr{R}, \mathscr{L})$. Then $\sigma\left([\lambda, \rho]\left[\lambda^{\prime}, \rho^{\prime}\right]\right)=\sigma\left(\left[\lambda \lambda^{\prime}, \rho \rho^{\prime}\right]\right)=\left[\lambda \lambda^{\prime}\right]=[\lambda]\left[\lambda^{\prime}\right]=\sigma([\lambda, \rho]) \sigma\left(\left[\lambda^{\prime}, \rho^{\prime}\right]\right)$ where $\lambda \lambda^{\prime}: D_{\lambda}^{\prime} \cap \lambda^{\prime-1} D_{\lambda} \rightarrow S$ and $\rho^{\prime} \rho: D_{\rho^{\prime}} \cap\left(\rho^{\prime}\right)^{-1} D_{\rho} \rightarrow S$. Thus $\sigma$ is a semigroup isomorphism.

(2) Similarly, if $\mathscr{R}$ is the collection of all right ideals of $S$, the mapping $\beta: \Omega(S: \mathscr{R}, \mathscr{L}) \rightarrow Q_{\mathscr{L}}(S)$ given by $\beta([\lambda, \rho])=[\rho]$ is a semigroup isomorphism from $\Omega(S: \mathscr{R}, \mathscr{L})$ onto the semigroup $Q_{\mathscr{L}}(S)$ of left quotients of $S$ developed by Hinkle [5].

(3) If $\mathscr{R}=\mathscr{L}=\{S\}$, then $\Omega(S: \mathscr{R}, \mathscr{L})=\Omega(S)$, the translational hull of $S$.

(4) Where $\mathscr{R}$ is the collection of all right ideals of $S$ and $\mathscr{L}$ is the collection of all left ideals of $S$, then $\Omega(K: \mathscr{R}, \mathscr{L})$ is trivial since $(\lambda, \rho) \Theta\left(\lambda^{\prime}, \rho^{\prime}\right)$ for all $\left(\lambda^{\prime}, \rho^{\prime}\right),(\lambda, \rho) \in B(K: \mathscr{R}, \mathscr{L})$ since $\lambda$ and $\lambda^{\prime}$ agree on $(0) \in \mathscr{R}$ and $\rho$ and $\rho^{\prime}$ agree on $(0) \in \mathscr{L}$.

(5) $\Omega(S: \mathscr{R}, \mathscr{L})$ always has an identity.

Proposition 2.5. $\Omega(K: \mathscr{R}, \mathscr{L})$ is an $(\mathscr{R}, \mathscr{L})$-extension of $\pi(K)$.

Proof. We will show that $\Omega(K: \mathscr{R}, \mathscr{L})$ is an $\mathscr{L}$-extension of $\pi(K)$. Since the $\mathscr{R}$-extension part is similar, it will be left to the reader.

Let $[\lambda, \rho] \in \Omega(K: \mathscr{R}, \mathscr{L})$ with $D_{\rho} \in \mathscr{L}$. For $s \in D_{\rho}, s[\lambda, \rho]=[s \lambda, s \rho]$. Now $D_{s \rho}=D_{\rho} s^{-1} \in \mathscr{L}$ and for $t \in D_{s \rho}, t(s \rho)=(t s) \rho$ and since $s \rho \in K$, $s \rho=\rho_{s \rho}$. On the other hand, for $t \in D_{s \lambda}=D_{\lambda}, s \lambda(t)=s(\lambda t)=(s \rho) t=$ $\lambda_{s \rho}(t)$. Since $s \rho \in K, s[\lambda, \rho]=\left[\lambda_{s \rho}, \rho_{s \rho}\right] \in \pi(K)$.

Corollary 2.6. When $K$ is $(\mathscr{R}, \mathscr{L})$-reductive, $\Omega(K: \mathscr{R}, \mathscr{L})$ is an $(\mathscr{R}, \mathscr{L})$-extension of $K$.

When $S$ is a semigroup, we are interested in the idealizer of $\pi(S)$ in $\Omega(S: \mathscr{R}, \mathscr{L})$.

Proposition 2.6. If $S$ is $(\mathscr{R}, \mathscr{L})$-reductive, then the idealizer of $\pi(S)$ in $\Omega(S: \mathscr{R}, \mathscr{L})$ is

$$
T=\left\{[\lambda, \rho]: D_{\lambda}=D_{\rho}=S\right\} .
$$

Proof. Note first that if $s \in D_{\lambda}$, and $(\lambda, \rho)$ are linked, then for 
$t \in s^{-1} D_{\lambda}$

$$
\lambda s(t)=\lambda(s t)=(\lambda s)(t)=\lambda_{\lambda s}(t)
$$

and for $t \in D_{\rho}$ we have

$$
(t)(\rho s)=(t \rho) s=t(\lambda s)=(t) \rho_{\lambda s} .
$$

Thus if $(\lambda, \rho) \in T, s \in D_{\lambda}$ we have

$$
(\lambda, \rho) \pi(s)=(\lambda, \rho)\left(\lambda_{s}, \rho_{s}\right)=(\lambda s, \rho s)=\left(\lambda_{\lambda s}, \rho_{\lambda s}\right)=\pi(\lambda s) .
$$

Similarly if $(\lambda, \rho)$ are linked and $s \in D_{\rho}$, then $(t) s \rho=(t) \rho_{s \rho}$ and $s \lambda(t)=\lambda_{s \rho}(t)$.

Thus

$$
\pi(s)(\lambda, \rho)=\pi(s \rho) \text { for }(\lambda, \rho) \in T .
$$

Therefore, $T$ is contained in the idealizer of $\pi(S)$.

Conversely, let $(\lambda, \rho)$ belong the idealizer of $\pi(S)$ in $\Omega(S: \mathscr{R}, \mathscr{L})$. Then for all $s \in S,(\lambda, \rho) \pi(s)$ and $\pi(s)(\lambda, \rho)$ belong to $\pi(S)$. We consider $(\lambda, \rho) \pi(s)$ since the other case is similar. Now $(\lambda, \rho) \pi(s)=$ $\pi(t)$ for some $t \in S$. Define $\lambda^{\prime}: S \rightarrow S$ by $\lambda^{\prime}(s)=t$ if $(\lambda, \rho) \pi(s)=\pi(t)$. Note that $\lambda^{\prime}$ is well defined since $\pi$ is injective. Moreover $\lambda^{\prime}$ agrees with $\lambda$ on $D_{\lambda}$ since if $s \in D_{\lambda},(\lambda, \rho) \pi(s)=\pi(\lambda s)$. It remains to show that $\lambda^{\prime}$ is a right $S$-homomorphism for then $(\lambda, \rho) \Theta\left(\lambda^{\prime}, \rho\right)$. Similarly define $\rho^{\prime}: S \rightarrow S$ by $(s) \rho^{\prime}=t$ iff $\pi(s)\left(\lambda^{\prime}, \rho\right)=\pi(s)(\lambda, \rho)=\pi(t)$. Then $(\lambda, \rho) \Theta\left(\lambda^{\prime}, \rho^{\prime}\right)$ and $\left(\lambda^{\prime}, \rho^{\prime}\right) \in T$.

Suppose $\lambda^{\prime}(s)=t$ and $\lambda^{\prime}(s x)=a$. We consider two cases. First, if $s \in D_{\lambda}$, then $\lambda^{\prime}(s)=\lambda s=t$ and $a=\lambda^{\prime}(s x)=\lambda(s x)=(\lambda s) x=t x$ and so $\lambda^{\prime}(s) x=\lambda^{\prime}(s x)$. Next, if $s \notin D_{\lambda}$, then for $z \in x^{-1} s^{-1} D_{\lambda}=(s x)^{-1} D_{\lambda}$,

$$
a z=(\lambda s x)[z]=\lambda[s(x z)]=\lambda s[x z]=t[x z]=(t x) z .
$$

Moreover, for $y \in D_{\rho}$

$$
y a=y(\rho s x)=[(y \rho) s] x=[y \rho s] x=(y t) x=y(t x)
$$

thus $\pi(a)=\pi(t x)$ or $a=t x$ or $\lambda^{\prime}(s x)=\lambda^{\prime}(s) x$.

Corollary 2.8. When $\mathscr{L}=\mathscr{R}=\{S\}$, and $S$ is $(\mathscr{R}, \mathscr{L})$ reductive then $\pi(S)$ is an ideal of $\Omega(S: \mathscr{R}, \mathscr{L})$.

3. Two sided semigroup of quotients. In the last section we show that $\Omega(S: \mathscr{R}, \mathscr{L})$ can be the translational hull or a semigroup of quotients of $S$. In this section we show that $\Omega(S: \mathscr{R}, \mathscr{L})$ can naturally be considered as a two-sided semigroup of quotients of $S$.

Definition 3.1. Let $V$ be an $(\mathscr{R}, \mathscr{L})$ extension of $K$. For each 
$a \in V$, define $\lambda^{a}, \rho^{a}, \tau^{a}=\left[\lambda^{a}, \rho^{a}\right]$ where $\lambda^{a}: a^{-1} K \rightarrow K$ is given by $\lambda^{a}(d)=a d$ and $\rho^{a}: K \mathrm{a}^{-1} \rightarrow K$ is given by $(d) \rho^{a}=d a$.

THEOREM 3.2. If $V$ is an $(\mathscr{R}, \mathscr{L})$ extension of $K$, the mapping $\tau: a \rightarrow \tau^{a}$ is a canonical $(S, S)$-homomorphism of $V$ into $\Omega(K: \mathscr{R}, \mathscr{L})$ which extends the canonical homomorphism $\pi$ of $K$ into $\Omega(K: \mathscr{R}, \mathscr{L})$.

The proof of the above theorem is straight forward and will be omitted. When there is any danger of confusion, we will denote $\tau: V \rightarrow \Omega(K: \mathscr{R}, \mathscr{L})$ by $\tau(V: K)$.

Definition 3.3. The $(\mathscr{R}, \mathscr{L})$-congruence on an $(S, S)$-set $M$ is denoted by $\eta_{M}$ and defined by

$$
\begin{gathered}
\eta_{M}=\{(m, n) \mid t m=t n \\
\qquad \text { for all } t \text { in some } L \in \mathscr{L} \text { and } m r=n r \text { for } \\
\text { all } r \text { in some } R \in \mathscr{R}\} .
\end{gathered}
$$

When the filters $\mathscr{R}$ and $\mathscr{L}$ are to be stressed, we write

$$
\eta_{M}=\eta(M: \mathscr{R}, \mathscr{L}) \text {. }
$$

Lemma 3.4. The $(\mathscr{R}, \mathscr{L})$ congruence on $K$ is $\pi \circ \pi^{-1}$ where $\pi$ is the canonical homomorphism of $K$ into $\Omega(K: \mathscr{R}, \mathscr{L})$.

Corollary 3.5. $K$ is $(\mathscr{R}, \mathscr{L})$-reductive iff $\eta_{K}$ is identity congruence.

In order to determine when $\tau$ is the unique homomorphism, extending $\pi$ we use the following item.

Lemma 3.6. If $K$ is $(\mathscr{R}, \mathscr{L})$-reductive or both $\mathscr{R}$ and $\mathscr{L}$ are idempotent, then the $(\mathscr{R}, \mathscr{L})$-congruence on $K$ is the identity.

Proof. Suppose $\omega, \omega^{\prime} \in \Omega(K: \mathscr{R}, \mathscr{L})$ and $\left(\omega, \omega^{\prime}\right) \in \eta_{\Omega(K: \mathscr{R}, €)}$ where $\omega=[\lambda, \rho]$ and $\omega^{\prime}=\left[\lambda^{\prime}, \rho^{\prime}\right]$. Then for all $x \in D_{\lambda} \cap D_{\lambda^{\prime}} \cap D$ and $s \in S$, (where $\omega x=\omega^{\prime} x$ for all $x \in D \in \mathscr{R}$ ),

$$
\lambda(x s)=\lambda \lambda_{x}(s)=\lambda^{\prime} \lambda_{x}(s)=\lambda^{\prime}(x s)
$$

and for all $y \in D_{\rho} \cap D_{\rho^{\prime}} \cap D^{\prime}$ and $s \in S$, (where $d \omega=d \omega^{\prime}$ for $d \in D^{\prime} \in \mathscr{L}$ )

$$
(s y) \rho=(s) \rho_{y} \rho=(s) \rho_{y} \rho^{\prime}=(s y) \rho^{\prime} \text {. }
$$

If $\mathscr{L}$ and $\mathscr{R}$ are idempotent, there is $B^{2} \in \mathscr{L}, A^{2} \in \mathscr{R}$ with $A^{2} \cong D_{\lambda} \cap D_{\lambda^{\prime}} \cap D$ and $B^{2} \subseteq D_{\rho} \cap D_{\rho^{\prime}} \cap D^{\prime}$ and so $\lambda$ and $\lambda^{\prime}$ agree on $A^{2}$ while $\rho$ and $\rho^{\prime}$ agree on $B^{2}$, thus $(\lambda, \rho) \Theta\left(\lambda^{\prime}, \rho^{\prime}\right)$ and $\omega=\omega^{\prime}$ in $\Omega(K: \mathscr{R}, \mathscr{L})$. 
On the other hand if $x \in D_{\lambda} \cap D_{\lambda^{\prime}} \cap D$ and $y \in D_{\rho} \cap D_{\rho^{\prime}} \cap D^{\prime}$, then

$$
y(\bar{\lambda} x)=(y \rho) x=(y) \rho \rho_{x}=(y) \rho^{\prime} \rho_{x}=\left(y \rho^{\prime}\right) x=y\left(\lambda^{\prime} x\right)
$$

and similarly $(y \rho) x=\left(y \rho^{\prime}\right) x$. Consequently if $K$ is $(\mathscr{R}, \mathscr{L})$-reductive, $y \rho=y \rho^{\prime}$ and $\lambda x=\lambda^{\prime} x$ for all $y \in D_{\rho} \cap D_{\rho^{\prime}}$ and $x \in D_{\lambda} \cap D_{\lambda^{\prime}}$ and so $(\lambda, \rho) \Theta\left(\lambda^{\prime}, \rho^{\prime}\right)$ and $\omega=\omega^{\prime}$.

Proposition 3.7. If $K$ is $(\mathscr{R}, \mathscr{L})$ reductive or both $\mathscr{R}$ and $\mathscr{L}$ are idempotent, then $\tau(V: K)$ is the unique $(S, S)$-homomorphism of $V$ into $\Omega(K: \mathscr{R}, \mathscr{L})$ extending $\pi$.

The proof of this proposition is a simple modification of the proof of Proposition 1.3 of [4] and so will be omitted.

Proposition 3.8. If $V$ and $V^{\prime}$ are $(\mathscr{R}, \mathscr{L})$-extensions of $K$ and $\phi$ is an $(S, S)$-homomorphism of $V$ into $V^{\prime}$ which is the identity on $K$, then

$$
\tau(V: K)=\tau\left(V^{\prime}: K\right) \circ \phi
$$

Proof. Let $v \in V, x \in v^{-1} K$ and $y \in K v^{-1}$, then $\phi(v) x=\phi(v x)=v x$ and $y \phi(v)=\phi(y v)=y v$. Thus $\rho_{\phi(v)}$ and $\rho_{v}$ agree on $K v^{-1} \in \mathscr{L}$ and $\lambda_{\rho(v)}$ and $\lambda_{v}$ agree on $v^{-1} K \in \mathscr{R}$ and so the conclusion follows.

For a right $S$-set $M$ and $r q f \mathscr{R}$, Hinkle [5] defined the $\mathscr{R}$ singular congruence $\eta(\mathscr{R})$ on $M$ by

$\eta(\mathscr{R})=\{(m, n) \mid m r=n r$ for all $r$ in some $R \in \mathscr{R}\}=\eta(M: R)$

Similarly for a left $S$-set $N$ and $l q f \mathscr{L}$, there is an $\mathscr{L}$-singular congruence $\eta(\mathscr{L})$ on $N . \quad \eta(\mathscr{R})$ is a right $S$-congruence and $\eta(\mathscr{L})$ is a left $S$-congruence.

Lemma 3.9. For an $(S, S)$ set $M$, rqf $\mathscr{R}$ and $l q f \mathscr{L}$,

$$
\eta(M: \mathscr{R}, \mathscr{L})=\eta(\mathscr{R}) \cap \eta(\mathscr{L}) .
$$

The proof is straightforward as is the proof of the next lemma and hence both proofs are omitted.

LEMMA 3.10. If one of $\eta(\mathscr{R})$ or $\eta(\mathscr{L})$ is the identity congruence, then $M$ is $(\mathscr{R}, \mathscr{L})$-reductive.

Given a right $S$-set $K$ and a $r q f \mathscr{R}$, Hinkle [5] constructed a maximal right $S$-set of quotients $Q(K: \mathscr{R})$ of $K$ as the $S$-set of all right $S$-homomorphisms with domain a member of $\mathscr{R}$ and codomain 
$K$ factored by the congruence $\lambda \Theta \lambda^{\prime}$ iff $\lambda s=\lambda^{\prime} s$ for all $s$ in some $R \cong D_{\lambda} \cap D_{\lambda^{\prime}}$ where $R \in \mathscr{R}$. An $S$-homomorphism $\lambda: K \rightarrow Q(K: \mathscr{R})$ can be defined by $\lambda(k)=\left[\lambda_{k}\right]$ where $\lambda_{k}: S \rightarrow K$ is given by $s \rightarrow k s$. Then $\lambda \circ \lambda^{-1}=\eta(K: \mathscr{R})$ and $\lambda$ is an injection of $K$ into $Q(K: \mathscr{R})$ when $\eta(K: \mathscr{R})$ is the identity. Analogous results hold for a left $S$-set $M$ and $l q f \mathscr{L}$. The maximal left $S$-set of quotients of $M$ is denoted by $Q(M: \mathscr{L})$.

Now let $S$ be a semigroup with zero, $\mathscr{L}$ be a $l q f$ on $S, \mathscr{R}$ be a $r q f$ on $S$ and both $\eta(S: \mathscr{L})$ and $\eta(S: \mathscr{R})$ be the identity. Note that $S$ is a right $S$-set, left $S$-set and $(S, S)$-set with respect to the semigroup multiplication. Since both $\eta(S: \mathscr{L})$ and $\eta(S: \mathscr{R})$ are the identity, $S$ is $(\mathscr{R}, \mathscr{L})$ reductive, and so we identify $S$ with $\pi(S) \subseteq$ $\Omega(S: \mathscr{R}, \mathscr{L}), \lambda(S) \subseteq Q(S: \mathscr{R})$ and $\rho(S) \subseteq Q(S: \mathscr{L})$.

Now $Q(S: \mathscr{R})$ is a semigroup under the multiplication $\left[\lambda_{1}\right]\left[\lambda_{2}\right]=$ $\left[\lambda_{1} \circ \lambda_{2}\right]$ where $\lambda_{1} \circ \lambda_{2}: \lambda_{2}^{-1}\left(D_{\lambda_{1}}\right) \rightarrow S$ is the composition map. Moreover the canonical map $\lambda: S \rightarrow Q(S: \mathscr{R})$ is a semigroup monomorphism. Let $V=\left\{q \in Q(S: \mathscr{R}) \mid S q^{-1} \in \mathscr{L}\right\}$. Then $V$ is the maximal subsemigroup of $Q(S: \mathscr{R})$ which is an $(\mathscr{R}, \mathscr{L})$ extension of $S$. Define $\phi: V \rightarrow Q(S: \mathscr{L})$ by $\phi(q)=q^{\prime}=\left[\rho^{q}\right]$. Thus $\phi$ is a semigroup homomorphism which is the identity on $S$ since $\rho^{q_{1} q_{2}}$ agrees with $\rho^{q_{1}} \circ \rho^{q_{2}}$ on $S q_{2}^{-1} q_{1}^{-1} \in \mathscr{L}$. Since $\phi$ is the identity on $S, \phi(s q)=s \dot{\phi}(q)=s q^{\prime}$ and so $\phi(V)=\left\{q^{\prime} \in Q(S: \mathscr{L}):\left(q^{\prime}\right)^{-1} S \in \mathscr{R}\right\}$. Since $\phi$ is a monomorphism, we identify $V$ with $\phi(V)$ and so $V=Q(S: \mathscr{R}) \cap Q(S: \mathscr{L})$.

Now $\tau(V: S)=\tau\left(V^{\prime}: S\right) \circ \phi$ by Proposition 3.8. Moreover, $\tau(V: S)$ is injective for if $\tau^{q_{1}}=\tau^{q_{2}}$, then $\left(\lambda^{q_{1}}, \rho^{q_{1}}\right) \Theta\left(\lambda^{q_{2}}, \rho^{q_{2}}\right)$ so there is $R \in \mathscr{R}$ with $q_{1} r=q_{2} r$ for all $r \in R$, thus $q_{1}=q_{2}$. Recall $\tau(V: S)$ is the identity on $S$.

Finally we show that $\tau(V: S)$ is surjective. Let $[\lambda, \rho] \epsilon$ $\Omega(S: \mathscr{R}, \mathscr{L})$, then $q=[\lambda] \in Q(S: \mathscr{R})$ and $q^{\prime}=[\rho] \in Q(S: \mathscr{L})$. It suffices to show that $q \in V$. To this end let $t \in D_{\rho}$, and $s \in D_{\lambda}$, then

$$
(t \rho) s=t(\lambda s)=t(q s)=(t q) s \in S .
$$

Thus $\lambda^{(t q)}=\lambda_{(t \rho)}$ on $D_{\lambda} \in \mathscr{R}$, and since $t \rho \in S$ for $t \in D_{\rho},\left[\lambda_{t \rho}\right]=\left[\lambda^{(t q)}\right]=$ $t q \in S$ for all $t \in D_{\rho}$. Thus $q \in V$. Clearly $\tau^{q}=\left[\lambda^{q}, \rho^{q}\right]=[\lambda, \rho]$ and so $\tau(V: S)$ is surjective.

We have proven the following result.

Theorem 3.11. When both $\eta(S: \mathscr{R})$ and $\eta(S: \mathscr{L})$ are the identity congruence, $\Omega(S: \mathscr{R}, \mathscr{L})$ is a semigroup isomorphic over $S$ to $V^{\prime}=\left\{q \in Q(S: \mathscr{L}) \mid q^{-1} S \in \mathscr{R}\right\}$ and $\left\{q \in Q(S: \mathscr{R}) \mid S q^{-1} \in \mathscr{L}\right\}=V$.

If we identify $V$ with $V^{\prime}$ and $\Omega(S: \mathscr{R}, \mathscr{L})$, the above result shows that $\Omega(S: \mathscr{R}, \mathscr{L})=Q(S: \mathscr{R}) \cap Q(S: \mathscr{L})$. 
4. Strict and pure extensions. In the remaining sections essential extensions will play a large role. However, we would like information on $(\mathscr{R}, \mathscr{L})$-extensions $V$ of $K$ which fail to be essential. We can classify such extensions by their image under $\tau(V: K)$.

Definition 4.1. The type of an $(\mathscr{R}, \mathscr{L})$-extension $V$ of $K$ is the image $T(V: K)$ of $V$ under $\tau(V: K)$.

When $K$ is $(\mathscr{R}, \mathscr{L})$-reductive, it is easily seen that the types of extensions correspond to the subsets $T$ of $\Omega(K: \mathscr{R}, \mathscr{L})$ which are $(S, S)$-sets containing $K$. We first discuss the $(\mathscr{R}, \mathscr{L})$-extensions $V$ of $K$ which are, in some sense, as bad as possible-that is, for $v \in V$, there is $k \in K$ for which $x k=x v$ for all $x$ in some member $L$ of $\mathscr{L}$ and $k y=v y$ for all $y$ in some $R \in \mathscr{R}$.

Definition 4.2. An $(\mathscr{R}, \mathscr{L})$-extension $V$ of $K$ is strict if it has type $\pi(K)$.

When $K$ is $(\mathscr{R}, \mathscr{L})$-reductive, we will characterize the strict $(\mathscr{R}, \mathscr{L}$ )-extensions of $K$ by means of (partial) homomorphisms.

REMARK. In this and the following actions we pretty much follow the approach of Grillet and Petrich [4]. The proofs of many of the results are easy modifications of the proofs in [4] and so will be omitted.

Definition 4.3. An $(S, S)$-set $T$ is $(\mathscr{R}, \mathscr{L})$-trivial if for each $t \in T, 0 t^{-1} \in \mathscr{L}$ and $t^{-1} 0 \in \mathscr{R}$.

An $(\mathscr{R}, \mathscr{L})$-extension $V$ of $K$ is called an $(\mathscr{R}, \mathscr{L})$-extension of $K$ by $T$ if $T$ is $(S, S)$ isomorphic to the factor $(S, S)$-set $V / K$. Notice that in this case $T$ is $(\mathscr{R}, \mathscr{L})$-trivial.

Strict $(\mathscr{R}, \mathscr{L})$-extensions of $(\mathscr{R}, \mathscr{L})$-reductive $S$-sets by $T$ may be characterized in terms of partial homomorphisms of $T$ in the following sense:

Definition 4.3. Let $V$ be an $(\mathscr{R}, \mathscr{L})$-extension of $K$ by $T$. The extension $V$ is said to be determined by a partial homomorphism $\pi: T \backslash\{0\} V$ if for nonzero $a \in T$ and all $s \in S$,

$$
a \circ s=\left\{\begin{array}{ccc}
a s & \text { if } & a s \neq 0 \\
\pi(a) s & \text { if } & a s=0
\end{array}\right.
$$

while

$$
s \circ a=\left\{\begin{array}{ccc}
s a & \text { if } & s a \neq 0 \\
s \pi(a) & \text { if } & s a=0
\end{array}\right.
$$


where $\circ$ is the scalar multiplication in $V$.

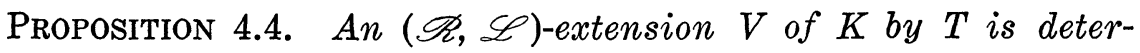
mined by a partial homomorphism iff $K$ is an $(S, S)$-retract of $V$.

The proof of Petrich ([9], Proposition 2, p. 51) carries over verbati to this case.

Proposition 4.5. Each $(\mathscr{R}, \mathscr{L})$-extension determined by a partial homomorphism is strict.

There is a converse to this proposition when $K$ is $(\mathscr{R}, \mathscr{L})$ reductive.

THEOREM 4.6. Let $K$ be $(\mathscr{R}, \mathscr{L})$-reductive. Then each strict $(\mathscr{R}, \mathscr{L})$-extension of $K$ is determined by a partial homomorphism.

Proof. Let $\tau=\tau(V: K)$ where $V$ is a strict extension of $K$. Then $\tau: V \rightarrow \pi(K)$ and since $\pi$ is an isomorphism, $\pi^{-1} \circ \tau: V \rightarrow K$ is an $(S, S)$-homomorphism whose restriction to $K$ is the identity.

COROLlaRY 4.7. Let $S$ be an $(\mathscr{R}, \mathscr{L})$-reductive semigroup and $Q$ be an $(\mathscr{R}, \mathscr{L})$-trivial semigroup. Then there is a strict $(\mathscr{R}, \mathscr{L})$ extension of $S$ by $Q$ iff there is a partial homomorphism of $Q \backslash\{0\}$ into $S$.

Strict $(\mathscr{R}, \mathscr{L})$-extensions of $K$ can be characterized as follows

Proposition 4.8. Let $V$ be an $(\mathscr{R}, \mathscr{L})$-extension of $K$. If any $(S, S)$-homomorphism of $K$ into another $(S, S)$-set can be extended to $V$, then $V$ is a strict extension of $K$. The converse holds if $K$ is $(\mathscr{R}, \mathscr{L})$-reductive.

Proof. The identity map id: $K \rightarrow K$ is an $(S, S)$-homomorphism and so has an extension $f: V \rightarrow K$. Thus $K$ is a retract of $V$ and so $V$ is a strict extension of $K$.

Conversely, let $K$ be $(\mathscr{R}, \mathscr{L})$-reductive and $K$ be a retract of $V$. Let $\alpha: K \rightarrow T$ be an $(S, S)$-homomorphism. Then if $r: V \rightarrow K$ is the retraction, $r \circ \alpha: V \rightarrow T$ is the desired extension.

Finally, strict extensions shed some light on the structure of $S$.

Proposition 4.9. If every $(\mathscr{R}, \mathscr{L})$-extension of $S$ is strict, then $S$ has an identity. 
Proof. The extension $S^{1}$ obtained by adjoining an identity to $S$ is an $(\mathscr{R}, \mathscr{L})$-extension since $S \in \mathscr{R} \cap \mathscr{L}$ and so is strict. Thus for some $c \in S, \tau^{1}=\pi c$. Thus for $x \in S$,

$$
1 x=c x=x=x c=x e
$$

since $D_{\lambda} 1=D_{\rho} 1=S$. Thus $c$ is an identity for $S$.

At the opposite end of the spectrum from the strict extensions, we have the pure extensions.

Definition 4.10. An $(\mathscr{R}, \mathscr{L})$-extension $V$ of $K$ is pure if the canonical homomorphism $v: V / K \rightarrow T(V: K) / \pi(K)$ satisfies $v^{-1}(0)=\{0\}$ where $v$ is induced by $\tau(V: K)$.

Lemma 4.11. An $(\mathscr{R}, \mathscr{L})$-extension $V$ of $K$ is pure iff for any $a \in V, \tau^{a} \in \pi(K)$ implies $a \in K$.

Lemma 4.11 says that pure extensions are "best" in the sense that no element of $V$ agrees with some element of $K$ on a member of $\mathscr{L}$ and on a member of $\mathscr{R}$.

We have the following result which determines all pure $(\mathscr{R}, \mathscr{L})$ extensions of $S$.

Definition 4.12. An $(S, S)$ homomorphism between $(S, S)$-sets with zero $f: K \rightarrow Q$ is pure if $f^{-1}(0)=\{0\}$.

THeOREM 4.13. Let $K$ be $(\mathscr{R}, \mathscr{L})$-reductive and $Q$ be an $(\mathscr{R}, \mathscr{L})$ trivial (S, S)-set with zero. Every pure homomorphism of $Q$ onto the $(S, S)$-set $T / \pi(K)$, where $T$ is a type of $(\mathscr{R}, \mathscr{L})$-extension of $K$, determines a pure $(\mathscr{R}, \mathscr{L})$-extension of $K$ by $Q$ of type $T$, whose scalar multiplication * is given by the following formula (where $Q^{*}=Q \backslash\{0\}$ and $\Theta(a)=\Theta^{a}=\left[\lambda^{a}, \rho^{a}\right] \in T \backslash \pi(K)$ for $\left.a \in Q^{*}\right)$ :

$$
a^{*} b=\left\{\begin{array}{cl}
a b & a \in K, b \in S \text { or } b \in K, a \in S \\
\Theta(a b)=\Theta^{a} b & a \in Q^{*}, b \in S \\
\Theta(a b)=a \Theta^{b} & a \in S, b \in Q^{*} .
\end{array}\right.
$$

Conversely, every pure extension of $K$ can be constructed in this fashion.

COROLlary 4.14. When $Q^{*}$ is a semigroup, $\Theta$ is a semigroup homomorphism and $K=S$, then the above result shows that each pure $(\mathscr{R}, \mathscr{L})$-extension can be given a semigroup multiplication by defining for $a, b \in Q^{*}, a^{*} b=a b$ if $a b \neq 0$ and $a^{*} b=s \in S$ if $a b=0$ and $\Theta^{a} \Theta^{b}=\pi s \in \pi(S)$. 
The proof of the above results is an easy modification of the proof of Theorem 2.11 of [4].

The reason for considering strict and pure extensions is evident by the next theorem.

THEOREM 4.15. Let $V$ be an $(\mathscr{R}, \mathscr{L})$-extension of $K$. The complete inverse image $U$ of $\pi(K)$ under $\tau(V: K)$ is the greatest strict $(\mathscr{R}, \mathscr{L})$-extension of $K$ in $V$ and $V$ is a pure $(\mathscr{R}, \mathscr{L})$-extension of $U$.

Proof. Since $\tau(V: K)$ is a homomorphism and $T(V: K)$ contains $\pi(K), U$ is an $(S, S)$-subset of $V$ and so is an $(\mathscr{R}, \mathscr{L})$-subset of $V$. Since $\tau(V: K)$ maps $K$ into $\pi(K)$, we must have $K \subseteq U$. And since $U \subseteq V, U$ is an $(\mathscr{R}, \mathscr{L})$-extension of $K$. Clearly $U$ is a strict extension of $K$. Moreover if $U^{\prime}$ is an $(\mathscr{R}, \mathscr{L})$-extension of $K$ in $V$, then $\tau\left(U^{\prime}: K\right)$ is the restriction of $\tau(V: K)$ to $U^{\prime}$. Hence if $U^{\prime}$ is strict, then $U^{\prime} \subseteq U$ and so $U$ is the greatest strict $(\mathscr{R}, \mathscr{L})$-extension of $K$ in $V$.

Now let $v \in V$, and suppose that $\tau^{v}(V: U) \in \pi(U)$. Then for some $u \in U, v s=u s$ for all $s \in R^{\prime} \in \mathscr{R}$ and $t v=t u$ for all $t \in L^{\prime} \in \mathscr{L}$. But $u \in U$ implies that for some $k \in K, k x=u x$ for all $x$ in some $R^{\prime \prime} \in \mathscr{R}$ and $y k=y u$ for $y \in$ some $L^{\prime \prime} \in \mathscr{L}$. Let $R=R^{\prime} \cap R^{\prime \prime} \in \mathscr{R}$ and $L=L^{\prime} \cap L^{\prime \prime} \in \mathscr{L}$, then for all $x \in L$ and all $y \in R, \quad x v=x k$ and $v y=k y$ and so $v \in U$.

5. Congruence dense extensions. In this section we will show for $(\mathscr{R}, \mathscr{L})$-reductive $K$, that $\Omega(K: \mathscr{R}, \mathscr{L})$ is the maximal essential $(\mathscr{R}, \mathscr{L})$-extension of $K$ and so is unique up to isomorphism.

In the remainder of this section, $V$ will be an $(\mathscr{R}, \mathscr{L})$-extension of $K$.

An $(S, S)$-congruence on $V$ whose restriction to $K$ is the identity is called a $K$-congruence.

$V$ is a congruence dense extension of $K$ if the identity is the only $K$-congruence on $V$.

$V$ is an essential extension of $K$ if each $(S, S)$-homomorphism $f: V \rightarrow T, T$ any $(S, S)$-set, whose restriction to $K$ is injective is an injection.

Berthiaume [1] has shown that congruence dense extensions coincide with essential extensions.

Lemma 5.1. $\eta(V: \mathscr{R}, \mathscr{L})=\tau \circ \tau^{-1}$ where $\tau=\tau(V: K)$.

Proof. $(x, y) \in \eta(V: \mathscr{R}, \mathscr{L})$ iff there is $R \in \mathscr{R}, L \in \mathscr{L}$ with $x r=y r$ for $r \in \mathscr{R}$ and $t x=t y$ for $t \in L$. Let $L^{\prime}=K x^{-1} \cap K v^{-1} \cap$ 
$L \in \mathscr{L}$ and $R^{\prime}=x^{-1} K \cap y^{-1} K \cap R \in \mathscr{R}$. Then $t x=t y$ for $t \in L^{\prime}$ and $x r=y r$ for $r \in R^{\prime}$, thus $\tau^{x}=[\lambda x, \rho x]=[\lambda y, \rho y]=\tau^{y}$.

Conversely if $\tau^{x}=\tau^{y}$, then $\left[\lambda^{x}, \rho^{x}\right]=\left[\lambda^{y}, \rho^{y}\right]$ so there is $L \in \mathscr{L}$ and $R \in \mathscr{R}$ with $x r=y r$ for $r \in \mathscr{R}$ and $t x=t y$ for $t \in L$. Thus $(x, y) \in \eta(V: \mathscr{R}, \mathscr{L})$.

THEOREM 5.2. Every $K$-congruence on $V$ is contained in $\eta(V: \mathscr{R}, \mathscr{L}) . \quad$ Moreover if $K$ is $(\mathscr{R}, \mathscr{L})$-reductive, $\eta(V: \mathscr{R}, \mathscr{L})$ is the largest $K$-congruence on $V$. In any case, $\eta(K)=\{v \in V \mid v \eta k$ for some $k \in K\}$ is the largest strict subextension of $V$.

Proof. Let $\mathscr{C}$ be a $K$-congruence on $V$. Then $a \mathscr{C} b$ implies $a s=b s$ for all $s \in a^{-1} K \cap b^{-1} K \in \mathscr{R}$. Likewise $t a=t b$ for all $t \in K a^{-1} \cap K b^{-1} \in \mathscr{L}$. Thus $(a, b) \in \eta(V: \mathscr{R}, \mathscr{L})$.

If $K$ is $(\mathscr{R}, \mathscr{L})$-reductive, then $\left.\eta(V: \mathscr{R}, \mathscr{L})\right|_{K}=\eta(K: \mathscr{R}, \mathscr{L})$ which is the identity thus $\eta(V: \mathscr{R}, \mathscr{L})$ is a $K$-congruence.

If $a \in \eta(K)$, then $\tau^{a}=\tau^{b}$ for some $k \in K$ and so $\eta(K)$ is the largest strict subextension of $V$.

Corollary 5.3. If $K$ is $(\mathscr{R}, \mathscr{L})$-reductive, then $V$ is a pure extension iff $\mathscr{C}(K)=K$ for every $K$-congruence on $V$.

When $K$ is $(\mathscr{R}, \mathscr{L})$-reductive, the following theorem characterizes strict $(\mathscr{R}, \mathscr{L})$-extensions by means of extensions of $(S, S)$ congruences on $K$. The proof is modelled after that of [4, Proposition 3.3].

THeOREM 5.4. Let $V$ be an $(\mathscr{R}, \mathscr{L})$-extension of $K$. If each $(S, S)$-congruence on $K$ is the restriction of some $(S, S)$-congruence $\overline{\mathscr{C}}$ on $V$ such that $\overline{\mathscr{C}}(K)=V$, then $V$ is a strict extension of $K$. The converse holds if $K$ is $(\mathscr{R}, \mathscr{L})$-reductive.

Proof. Let $\mathscr{C}$ be the identity congruence on $K$, then $\overline{\mathscr{C}}(K)=V$ and so for $v \in V$, there is a unique $k \in K$ with $v \overline{\mathscr{C}} k$. Now if $s \in K v^{-1}$, $s v \overline{\mathscr{C}} s k$ and so $s v=s k$ on $K v^{-1}$ and similarly, if $t \in v^{-1} K, v t \overline{\mathscr{C}} k t$ and so $v t=k t$ on $v^{-1} K$. These equations imply that $\tau^{v}=\tau^{k}$ and thus the extension is strict.

If $K$ is $(\mathscr{R}, \mathscr{L})$-reductive, then $K$ is a retract of $V$ iff $V$ is a strict extension of $K$. Then given an $(S, S)$-congruence $\mathscr{C}$ on $K$, extend $\mathscr{C}$ to $\overline{\mathscr{C}}$ on $V$ by $\omega \overline{\mathscr{C}} v$ iff $r(\omega) \mathscr{C} r(v)$ where $r: V \rightarrow K$ is the retraction. Then since $\omega \overline{\mathscr{C}} r(\omega), \overline{\mathscr{C}}(K)=V$.

REMARK. This result may be used to give a different proof of Proposition 4.8 . 
Theorem 5.2 characterizes congruence dense extensions of $(\mathscr{R}, \mathscr{L})$-reductive $(S, S)$-sets as follows:

THEOREM 5.5. Let $K$ be $(\mathscr{R}, \mathscr{L})$-reductive, then $V$ is congruence dense (essential) over $K$ iff $V$ is $(\mathscr{R}, \mathscr{L})$-reductive. Thus congruence dense extensions are pure.

When $\mathscr{L}$ is the lattice of left ideals of $S$, we have the following corollary due to Hinkle [5, Corollary 4.13].

COROLlaRY 5.6. When $S$ is $\mathscr{R}$-torsion free, then an $\mathscr{R}$-extension $V$ of $S$ is essential iff $V$ is $\mathscr{R}$-torsion free.

When $\mathscr{R}=\mathscr{L}=\{S\}$, we have the following corollary due to Petrich and Grillet [4, Theorem 3.7].

CoRollary 5.7. Let $S$ be weakly reductive. Then $V$ is a congruence dense extension of $S$ iff $\tau(V: S)$ is injective.

Returning to the general case we have the

Corollary 5.8. When $K$ is $(\mathscr{R}, \mathscr{L})$-reductive, $V$ is a congruence dense (essential) extension of $K$ iff there is a monomorphism of $V$ over $K$ into $\Omega(K: \mathscr{R}, \mathscr{L})$.

Since $\tau(\Omega: K)$ is the identity on $\Omega(K: \mathscr{R}, \mathscr{L})$ where $K$ is $(\mathscr{R}, \mathscr{L})$ reductive, then $\Omega(K: \mathscr{R}, \mathscr{L})$ is congruence dense over $K$. Hence when $\mathscr{L}$ is the lattice of left ideals of $S$ and $S$ is $\mathscr{R}$-torsion free, then [5, Corollary 5.6] $Q_{\mathscr{R}}(S)$ is essential over $S$.

Finally, we characterize pure $(\mathscr{R}, \mathscr{L})$-extensions of $(\mathscr{R}, \mathscr{L})$ reductive $(S, S)$-sets by means of congruence dense extensions as follows:

Corollary 5.9. Let $K$ be $(\mathscr{R}, \mathscr{L})$-reductive. Then $V$ is a pure extension iff there is an $(S, S)$-homomorphism $\dot{\rho}$ over $K$ of $V$ into a congruence dense extension $D$ of $K$ with $\phi^{-1}(K)=K$.

Proof. Let $T=T(V: K)$. Then by Corollaries 5.7 and 5.8, there is a dense extension $D$ of $K$ of type $T$. Then $\phi=\tau(D: K)^{-1} \circ \tau(V: K)$ is an $(S, S)$-homomorphism over $K$ of $V$ into $D$. Since $V$ is pure over $K, \phi^{-1}(K)=\tau(V: K)^{-1}(\pi(K))=K$.

Conversely, let $\phi$ be the given homomorphism, then $\tau(V: K)=$ $\tau(D: K) \circ \phi$ by 3.8 and $\tau(V: K)^{-1}(\pi(K))=\phi^{-1}(K)=K$ since $\tau(D: K)$ is injective. Thus $V$ is pure. 
We now prove the main result of this section.

THEOREM 5.10. When $K$ is $(\mathscr{R}, \mathscr{L})$-reductive, $\Omega(K: \mathscr{R}, \mathscr{L})$ is the maximal essential $(\mathscr{R}, \mathscr{L})$-extension of $K$.

Proof. By the remark after Corollary 5.8, $\Omega=\Omega(K: \mathscr{R}, \mathscr{L})$ is essential over $K$. Now suppose $V \supseteqq \Omega$ is an essential $(\mathscr{R}, \mathscr{L}$ )extension of $K$. Thus $\tau(V: K)$ is injective and $\left.\tau(V: K)\right|_{2}=\tau(\Omega: K)$ is the identity. Moreover if $v \notin \Omega$, then $\tau(V: K)(v)=x \in \Omega$, and $\tau(V: K)$ is injective. For $s \in \Omega v^{-1} \in \mathscr{L}$ and $t \in v^{-1} \Omega \in \mathscr{R}$, we have $x s=$ $\tau(V: K)(v s)=v s$ and $t x=\tau(V: K)(t v)=t v$. Thus $(x, v) \in \eta(V: \mathscr{R}, \mathscr{L})$ which is the identity. Thus $x=v$ and $V=\Omega(K: \mathscr{R}, \mathscr{L})$.

THEOREM 5.11. $\Omega(K: \mathscr{R}, \mathscr{L})$ is unique up to isomorphism over $K$, when $K$ is $(\mathscr{R}, \mathscr{L})$-reductive.

Proof. Let $V$ be any other maximal essential $(\mathscr{R}, \mathscr{L})$-extension of $K$. Then $\tau(V: K)$ is injective by Theorem 5.5. If $T(V: K)=$ $T \subseteq \Omega(K: \mathscr{R}, \mathscr{L})$, then $\tau(V: K)$ can be extended to an $(S, S)$-isomorphism of an $(\mathscr{R}, \mathscr{L})$-extension $V^{\prime} \supseteqq V$ onto $\Omega(K: \mathscr{R}, \mathscr{L})$. Consequently, $V^{\prime}$ is congruence dense over $K$ by Theorem 5.5. Thus $V^{\prime}=V$ and so $\tau(V: K)$ is an isomorphism.

When $\mathscr{R}=\mathscr{L}=\{S\}$, then we have as a corollary the following theorem of Gluskin [3]:

THEOREM 5.12. Let $S$ be weakly reductive, then $S$ is a densely embedded ideal of $V$ iff there is an isomorphism over $S$ of $V$ onto $\Omega(S)$.

Notice also that Theorem 5.10 says that $\Omega(S: \mathscr{R}, \mathscr{L})$ is not only the maximal congruence dense $(\mathscr{R}, \mathscr{L})$-semigroup extension but is also maximal among congruence dense $(\mathscr{R}, \mathscr{L})$-extensions as an $(S, S)$-set.

6. The injectivity of $\Omega(K: \mathscr{R}, \mathscr{L})$. In this section we show that $\Omega(K: \mathscr{R}, \mathscr{L})$ is the $(\mathscr{R}, \mathscr{L})$-injective hull of $K$. First we prove that an $(\mathscr{R}, \mathscr{L})$-injective hull of $K$ exists.

Definition 6.1. A bi-S-set ${ }_{S} K_{S}$ is $(\mathscr{R}, \mathscr{L})$-injective iff each $(S, S)$-homomorphism $f:{ }_{S} T_{S} \rightarrow{ }_{S} K_{S}$ has for any $(\mathscr{R}, \mathscr{L})$-extension ${ }_{S} N_{S}$ of $T$ an $(S, S)$-extension $\bar{f}: N \rightarrow K$. In particular, ${ }_{S} K_{S}$ is injective when $\mathscr{R}$ consists of all right ideals of $S$ and $\mathscr{L}$ consists of all left ideals.

Let $K$ be any bi-S-set and let $K^{S^{1}}$ denote the set of all mappings 
from $S^{1}$ to $K . \quad K^{S^{1}}$ is a bi-S-set under the mutiplication $(s f)(x)=$ $s(f(x))$ and $(f t)(x)=f(t x)$ for all $x \in S^{1}$. Consider $K$ as a subset of $K^{S^{1}}$ by $k: S^{1} \rightarrow K$ by $k(x)=k x$ for all $x \in S^{1}$. That $K^{S^{1}}$ is an injective $(S, S)$-set follows by noting in Theorem 6 of [1] that the constructed extension is an $(S, S)$-homomorphism.

Proposition 6.2. For each $(S, S)$-set $K$, there is an injective $(S, S)$-set $I_{S}$ containing $K$.

We require the following lemma from [1].

Lemma 6.3. Let $A, B$ and $C$ be $(S, S)$-sets with $A \leqq B \leqq C$. Then $A$ is essential in $C$ iff $A$ is essential in $B$ and $B$ is essential in $C$.

Now let ${ }_{S} K_{S}$ be given. Following Berthiaume, we see that $K$ has a maximal essential extension $\hat{K}$ which is also the minimal injective extension of $K$. Moreover $\hat{K}$ is unique up to isomorphism over $K$. For any injective extension $I$ of $K, \hat{K}$ is the maximal essential extension of $K$ in $I$. Let $E$ be a maximal $(\mathscr{R}, \mathscr{L}$ )-extension of $K$ in $\hat{K}$ which exists by Zorn's lemma.

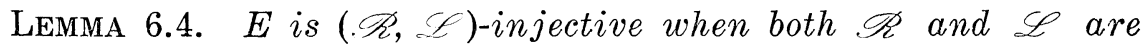
idempotent.

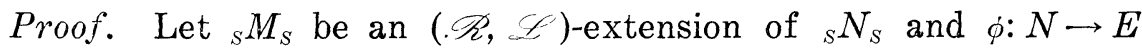
be an $(S, S)$-homomorphism. Let $\bar{\phi}: M \rightarrow \hat{K}$ be an extension of $\phi$ to $M$. Consider $W=\bar{\phi}(M) \cup E \cong \hat{K}$. It suffice to show that $W$ is an $(\mathscr{R}, \mathscr{C})$-extension of $E$, for then $W$ is an $(\mathscr{R}, \mathscr{L})$-extension of $K$ since $\mathscr{R}$ and $\mathscr{L}$ are idempotent; thus $W=E$ and we are done.

Therefore, let $\bar{\phi}(t) \notin E$. Then there is $R \in \mathscr{R}$ and $L \in \mathscr{L}$ with $t R \cong N$ and $L t \subseteq N$. Thus $\bar{\phi}(t) R=\bar{\phi}(t R) \subseteq E$ and likewise $L \bar{\phi}(t) \subseteq E$. Hence $W$ is an $(\mathscr{R}, \mathscr{L})$-extension of $E$ and we are done.

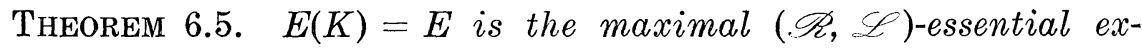
tension of $K$.

Proof. Let $T \supseteqq E$ be an $(\mathscr{R}, \mathscr{L})$-essential extension of $K$. Then without loss of generality, $T \subseteq K$, the maximal essential extension of $K$. Thus $T=E$.

Theorem 6.6. $E(K)=E$ is the minimal $(\mathscr{R}, \mathscr{L})$-injective extension of $K$, when both $\mathscr{R}$ and $\mathscr{L}$ are idempotent. 
Proof. Let $K \subseteq T \subseteq E$ and $T$ be an $(\mathscr{R}, \mathscr{L})$-injective extension of $K$. Then there is an extension $\phi: E \rightarrow T$ of the identity map 1: $T \rightarrow T$. By Lemma 6.3, $T$ is essential in $E$ and so $\phi$ is one-toone. Hence $T=E$.

By Theorem 5.10 when $K$ is $(\mathscr{R}, \mathscr{L})$-reductive, $\Omega(K: \mathscr{R}, \mathscr{L})$ is the maximal essential $(\mathscr{R}, \mathscr{L}$ )-extension of $K$. By Theorem 6.5 and Theorem 5.11, $\Omega(K: \mathscr{R}, \mathscr{L})$ is isomorphic over $K$ to $E$. Consequently, when $\mathscr{R}$ and $\mathscr{L}$ are idempotent, $\Omega(K: \mathscr{R}, \mathscr{L})$ is the injective hull of $K$ by Theorem 6.6. Thus we have proved the following theorem.

THEOREM 6.7. When $K$ is $(\mathscr{R}, \mathscr{L})$-reductive and both $\mathscr{R}$ and $\mathscr{L}$ are idempotent, the $\Omega(K: \mathscr{R}, \mathscr{L})$ is the $(\mathscr{R}, \mathscr{L})$-injective hull of $K$.

CoRollary 6.8. When $S$ is idempotent and weakly reductive, $\Omega(S)$ is $(\{S\},\{S\})$-injective.

Proof. Since $\mathscr{R}=\mathscr{L}=\{S\}$ and $S^{2}=S, \mathscr{R}$ and $\mathscr{L}$ are idempotent. The weak reductivity of $S$ implies that $\eta_{Q(S:\langle S\},\{S\})}$ is the identity and so $S$ is $(\mathscr{R}, \mathscr{L})$-reductive. The result now follows from Theorem 6.7.

7. An application. In this section, we apply our theory to show that when $S$ is weakly reductive, then $\Omega(S)=\Omega\left(S^{n}\right)$ for all $n$ positive.

Let $\mathscr{R}=\mathscr{L}=\{S\}$, and write $\Omega(K: \mathscr{R}, \mathscr{C})=\Omega(K: S, S)$.

Lemma 7.1. If $\eta(S: S, S)=\mathrm{id}$, then $\eta\left(S^{n}: S^{n}, S^{n}\right)=i$ for all $n$.

Proof. Let $x \neq y$ in $S^{n}$. Then $x \neq y$ in $S$ so there is $s_{1}, t_{1} \in S$ with $s_{1} x \neq s_{1} y$ and $x t_{1} \neq y t_{1}$. Now suppose we have $s_{1}, \cdots, s_{n-1}$, $t_{1}, \cdots, t_{n-1} \in S$ with $s_{n-1} \cdots s_{1} x \neq s_{n-1} \cdots \dot{s}_{1} y$ and $x t_{1} \cdots t_{n-1} \neq$ $y t_{1} \cdots t_{m-1}$. Then there is $s_{n}, t_{n} \in S$ with $s_{n} s_{n-1} \cdots s_{1} x \neq s_{n} s_{n-1} \cdots s_{1} y$ and $x t_{1} \cdots t_{n-1} \neq y t_{1} \cdots t_{n-1} t_{n}$. Since $t_{1} \cdots t_{n}, s_{n} \cdots s_{1} \in S^{n}$, the result follows.

Corollary 7.2. If $\eta(S: S, S)=\mathrm{id}$, then $\eta\left(S^{n}: S, S\right)=\mathrm{id}$.

Definition 7.3. ${ }_{S} K_{S}$ is strictly essential in ${ }_{S} N_{S}$ if for all $m \neq n$ in $N$, there are $s \in m^{-1} K \cap n^{-1} K$ and $t \in K m^{-1} \cap K n^{-1}$ with $m s \neq n s$ and $t m \neq t n$.

LemMa 7.4. [1]. If ${ }_{S} K_{S}$ is strictly essential in ${ }_{s} N_{S}$, then $K$ is essential in $N$. 
Lemma 7.5. For all positive $n,{ }_{s} S_{s}^{n}$ is strictly essential in ${ }_{s} S_{S}$ when $\eta(S: S, S)=$ id.

The proof of this lemma is contained in the proof of Lemma 7.1.

Now if $\eta(S: S, S)=$ id, i.e., if $S$ is weakly reductive, then ${ }_{S} S_{S}$ is essential over ${ }_{S} S_{S}^{n}$ and so $\tau\left(S, S^{n}\right)$ is injective. Thus without loss of generality $S^{n} \subseteq S \subseteq \Omega\left(S^{n}: S, S\right)$. Since $\Omega\left(S^{n}: S, S\right)$ is congruence dense over $S, S \subseteq \Omega\left(S^{n}: S, S\right) \subseteq \Omega(S: S, S)$ without loss of generality. By Lemma 6.3, $S^{n}$ is congruence dense in $\Omega(S: S, S)$ but since $\Omega\left(S^{n}: S, S\right)$ is the maximal congruence dense extension of $S^{n}, \Omega\left(S^{n}: S, S\right)=$ $\Omega(S: S, S)$.

THEOREM 7.6. Let $S$ be weakly reductive, then for $n>0$, $\Omega\left(S^{n}: S, S\right)=\Omega(S: S, S)$.

Next notice that $\Omega\left(S^{n}: S^{n}, S^{n}\right)$ is strictly $\left(S^{n}, S^{n}\right)$-essential over $S^{n}$ since $\eta\left(S^{n}: S^{n}, S^{n}\right)=$ id. Thus $\Omega\left(S^{n}: S^{n}, S^{n}\right)$ is strictly $(S, S)$-essential over $S^{n}$ since $S^{n} \leqq S$. Thus we may suppose $S^{n} \leqq \Omega\left(S^{n}: S^{n}, S^{n}\right) \subseteq$ $\Omega\left(S^{n}: S, S\right)$. However for $q_{1} \neq q_{2}$ in $\Omega\left(S^{n}: S, S\right)$, there are $s, t \in S$ with $s q_{1} \neq s q_{2}, q_{1} t \neq q_{2} t$ and $s q_{1}, s q_{2}, q_{1} t, q_{2} t \in S^{n}$. Since $\eta\left(S^{n}: S^{n}, S^{n}\right)=\mathrm{id}$, there is $s^{1}, t^{1} \in S^{n}$ with $s^{1} s q_{1} \neq s^{1} s q_{2}$ and $q_{1} t t^{1} \neq q_{2} t t^{1}$. Since $t t^{1}, s^{1} s \in S^{n+1} \cong S^{n}$, we see that $\Omega\left(S^{n}: S, S\right)$ is strictly $\left(S^{n}, S^{n}\right)$-essential over $S^{n}$. Hence it is $\left(S^{n}, S^{n}\right)$-essential and so $\left(S^{n}, S^{n}\right)$-congruence dense over $S^{n}$. Thus

$$
\Omega\left(S^{n}: S^{n}, S^{n}\right)=\Omega\left(S^{n}: S, S\right) .
$$

THEOREM 7.7. Let $S$ be weakly reductive. Then for all $n>0$, $\Omega\left(S^{n}\right)=\Omega(S)$.

Proof. The result follows from the above discussion upon noting that $\Omega\left(S^{n}\right)=\Omega\left(S^{n}: S^{n}, S^{n}\right)=\Omega\left(S^{n}: S, S\right)=\Omega(S: S, S)=\Omega(S)$.

REMARK. When $S$ is not weakly reductive, the above result is false. To see this let $S$ be a semigroup with zero satisfying $S^{n}=0$, $S^{n-1} \neq 0$. Then for $x \in S^{n-1}, \lambda_{x}: S \rightarrow S^{n-1}$ and $\rho_{x}: S \rightarrow S^{n-1}$ are the zero maps. Thus $\Omega\left(S^{n-1}: S, S\right)=0$, but $\Omega(S) \neq 0$ for there is $x \in S^{n-2}$ with $x S=0$.

\section{REFERENCES}

1. P. Berthiaume, The injective envelope of S-sets, Canad. Math. Bull., 10 (1967), 261-273.

2. E. H. Feller and R. L. Gantos, Completely injective semigroups, Pacific J. Math., 31 (1969), 359-366.

3. L. M. Gluskin, Ideals of semigroups, Math. Sbornik, 55 (1961), 421-448 (Russian). 
4. P. Grillet and M. Petrich, Ideal extensions of semigroups, Pacific J. Math., 26 (1968), 493-508.

5. C. V. Hinkle, Generalized semigroups of quotients, Trans. Amer. Math. Soc., 183 (1973), 87-117.

6. John K. Luedeman, A generalization of the concept of a ring of quotients, Canad. Math. Bull., 14 (1971), 517-529.

7. F. R. McMorris, On quotient semigroups, J. of Math. Sci., 7 (1972), 48-56.

8. - The singular congruence and the maximal quotient semigroup, Canad. Math. Bull., 15 (1972), 301-303.

9. M. Petrich, On extensions of semigroups determined by partial homomorphisms, Indag. Math., 28 (1966), 49-51.

10. - Introduction to Semigroups, Merrill, Columbus, Ohio, 1973.

Received June 11, 1975 and in revised form April 15, 1976.

Clemson University 



\section{PACIFIC JOURNAL OF MATHEMATICS}

\section{EDITORS}

RICHARD ARENS (Managing Editor)

University of California

Los Angeles, California 90024

\section{J. DugunduI}

Department of Mathematics University of Southern California Los Angeles, California 90007

D. Gilbarg and J. Milgram

Stanford University

Stanford, California 94305

\author{
R. A. Beaumont \\ University of Washington \\ Seattle, Washington 98105
}

\section{ASSOCIATE EDITORS}
B. H. NeUmanN
F. WOLF
K. YosHIDA

\section{SUPPORTING INSTITUTIONS}

\author{
UNIVERSITY OF BRITISH COLUMBIA \\ CALIFORNIA INSTITUTE OF TECHNOLOGY \\ UNIVERSITY OF CALIFORNIA \\ MONTANA STATE UNIVERSITY \\ UNIVERSITY OF NEVADA \\ NEW MEXICO STATE UNIVERSITY \\ OREGON STATE UNIVERSITY \\ UNIVERSITY OF OREGON \\ OSAKA UNIVERSITY
}

\author{
UNIVERSITY OF SOUTHERN CALIFORNIA \\ STANFORD UNIVERSITY \\ UNIVERSITY OF HAWAII \\ UNIVERSITY OF TOKYO \\ UNIVERSITY OF UTAH \\ WASHINGTON STATE UNIVERSITY \\ UNIVERSITY OF WASHINGTON \\ AMERICAN MATHEMATICAL SOCIETY
}

The Supporting Institutions listed above contribute to the cost of publication of this Journal, but they are not owners or publishers and have no responsibility for its content or policies.

Mathematical papers intended for publication in the Pacific Journal of Mathematics should be in typed form or offset-reproduced, (not dittoed), double spaced with large margins. Please do not use built up fractions in the text of your manuscript. You may however, use them in the displayed equations. Underline Greek letters in red, German in green, and script in blue. The first paragraph or two must be capable of being used separately as a synopsis of the entire paper. Items of the bibliography should not be cited there unless absolutely necessary, in which case they must be identified by author and Journal, rather than by item number. Manuscripts, in triplicate, may be sent to any one of the editors. Please classify according to the scheme of Math. Reviews, Index to Vol. 39. All other communications should be addressed to the managing editor, or Elaine Barth, University of California, Los Angeles, California, 90024.

The Pacific Journal of Mathematics expects the author's institution to pay page charges, and reserves the right to delay publication for nonpayment of charges in case of financial emergency.

100 reprints are provided free for each article, only if page charges have been substantially paid. Additional copies may be obtained at cost in multiples of 50 .

The Pacific Journal of Mathematics is issued monthly as of January 1966. Regular subscription rate: $\$ 72.00$ a year (6 Vols., 12 issues). Special rate: $\$ 36.00$ a year to individual members of supporting institutions.

Subscriptions, orders for back numbers, and changes of address should be sent to Pacific Journal of Mathematics, 103 Highland Boulevard, Berkeley, California, 94708.

PUBLISHED BY PACIFIC JOURNAL OF MATHEMATICS, A NON-PROFIT CORPORATION

Printed at Kokusai Bunken Insatsusha (International Academic Printing Co., Ltd.), 8-8, 3-chome, Takadanobaba, Shinjuku-ku, Tokyo 160, Japan. 


\section{Pacific Journal of Mathematics}

Patricia Andresen and Marvin David Marcus, Weyl's inequality and

quadratic forms on the Grassmannian .......................

George Bachman and Alan Sultan, Regular lattice measures: mappings and

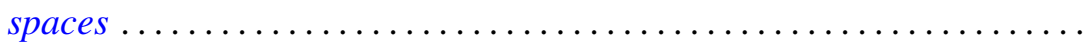

David Geoffrey Cantor, On certain algebraic integers and approximation by rational functions with integral coefficients ...................

James Richard Choike, On the value distribution of functions meromorphic in the unit disk with a spiral asymptotic value ..................

David Earl Dobbs, Divided rings and going-down................ 353

Mark Finkelstein and Robert James Whitley, Integrals of continuous

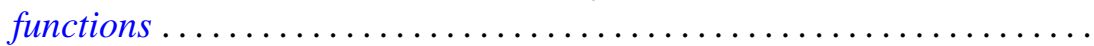

Ronald Owen Fulp and Joe Alton Marlin, Integrals of foliations on manifolds with a generalized symplectic structure ...............

Cheong Seng Hoo, Principal and induced fibrations .................

Wu-Chung Hsiang and Richard W. Sharpe, Parametrized surgery and

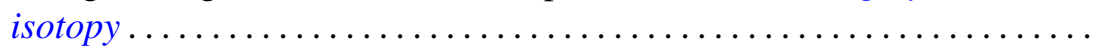

Surender Kumar Jain, Surjeet Singh and Robin Gregory Symonds, Rings whose proper cyclic modules are quasi-injective .................

Pushpa Juneja, On extreme points of the joint numerical range of commuting normal operators...

Athanassios G. Kartsatos, Nth order oscillations with middle terms of order $N-2$.

John Keith Luedeman, The generalized translational hull of a

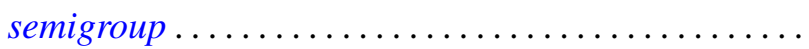

Louis Jackson Ratliff, Jr., The altitude formula and DVR's ...

Ralph Gordon Stanton, C. Sudler and Hugh C. Williams, An upper bound for the period of the simple continued fraction for $\sqrt{D}$...

David Westreich, Global analysis and periodic solutions of second order systems of nonlinear differential equations...

David Lee Armacost, Correction to: "Compactly cogenerated LCA

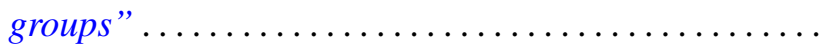

Jerry Malzan, Corrections to: "On groups with a single involution" .

David Westreich, Correction to: "Bifurcation of operator equations with unbounded linearized part" ...................... 